\title{
Un trono dividido: El origen y los movimientos de la Banca Jeroglífica 1 de Ixtutz, Guatemala
}

\author{
Nicholas Carter (D, Mara Antonieta Reyes, David Stuart, Stephen Houston, Katharine Lukach \\ y Megan O’Neil
}

\begin{abstract}
Aquí presentamos el descubrimiento de una banca jeroglífica esculpida del sitio maya de Ixtutz, Petén, Guatemala. Analizamos la inscripción jeroglífica en la banca y discutimos la historia del objecto en el contexto de su producción, su desmantelamiento y reutilización subsecuente, y la remoción de unas piezas del sitio. Mostramos que dos bloques inscritos de caliza, uno de ellos en una colección privada en Bruselas y el otro en el Los Angeles County Museum of Art, Los Angeles, California, pertenecen al mismo trono, y llamamos para su repatriación voluntaria a Guatemala.
\end{abstract}

Palabras clave: arqueología maya, epigrafía, repatriación, Guatemala, historia del arte

We present here the discovery of a sculptured hieroglyphic throne from the Maya site of Ixtutz, Petén, Guatemala. We analyze the hieroglyphic inscription on the throne and discuss the history of the object in the context of its production, its subsequent dismantling and reuse, and the removal of some pieces from the site. We show that two inscribed limestone blocks, one in a private collection in Brussels and the other in the Los Angeles County Museum of Art, Los Angeles, California, belong to the same throne and call for their voluntary repatriation to Guatemala.

Keywords: Maya archaeology, epigraphy, repatriation, Guatemala, art history

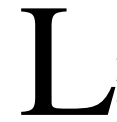

os artefactos arqueológicos no son simplemente instantáneas de los momentos pasados cuando fueron creados. Desde su producción, han sido descartados, abandonados, sujetos a la intemperie y la bioturbación. En la antigüedad pueden haber sido movidos, reutilizados, modificados; en la modernidad, han sido descubiertos - legítima o ilegalmenteapropiados, a veces vandalizados o "retocados", $\mathrm{y}$ han sido imbuidos de nuevos significados como símbolos nacionales, herencia cultural o arte de valor universal. Y en el camino pueden haber sido divididos y separados, divorciados de sus contextos originales y desmembrados. La pérdida de información en ese proceso puede ser tremenda.

Tal es el caso de una banca de múltiples bloques de piedra caliza con una inscripción jeroglífica maya, esculpido en el sitio de Ixtutz, Petén, Guatemala, al fin del período Clásico tardío (600-830 dC). Originalmente parte de un trono o una banca comisionada por un rey del sitio, la banca fue desmantelada y reutilizada durante el período Clásico terminal (830-900 dC). Tres

\footnotetext{
Nicholas Carter (npcarter@txstate.edu, autor de contacto) — Department of Anthropology, Texas State University, San Marcos, Texas, USA

Mara Antonieta Reyes - Centro Universitario de Petén, Universidad de San Carlos de Guatemala, Flores, Guatemala David Stuart - Department of Art and Art History, University of Texas, Austin, Texas, USA

Stephen Houston $\mathbf{a}$ Department of Anthropology, Brown University, Providence, Rhode Island, USA

Katharine Lukach $\mathbf{E}$ Escolar independiente, San Marcos, Texas, USA

Megan O'Neil Department of Art History, Emory University, Atlanta, Georgia, USA
}

Latin American Antiquity 33(2), 2022, pp. 262-278

Copyright (C) The Author(s), 2021. Published by Cambridge University Press on behalf of the Society for American Archaeology. This is an Open Access article, distributed under the terms of the Creative Commons Attribution licence (https:// creativecommons.org/licenses/by/4.0/), which permits unrestricted re-use, distribution, and reproduction in any medium, provided the original work is properly cited. doi:10.1017/laq.2021.76 
fragmentos del monumento se descubrieron en excavaciones por Mara Antonieta Reyes, directora del proyecto Atlas Arqueológico de Guatemala (AAG), en 2017 y 2018. En este artículo reunimos virtualmente estos fragmentos con otras dos piezas del mismo trono - una en una colección privada en Bruselas, Bélgica, y la otra en el Los Angeles County Museum of Art, Los Angeles, California (LACMA). Presentamos un dibujo de reconstrucción del trono, que llamamos la Banca Jeroglífica 1 de Ixtutz (o "Banca 1"); reconstruimos la historia del objecto a la medida de lo posible; y expresamos nuestra esperanza por la repatriación voluntaria de los dos bloques enajenados a la República de Guatemala.

\section{Contexto arqueológico e historia de investigaciones}

El sitio de Ixtutz se localiza en las Montañas Mayas, a $8 \mathrm{~km}$ al suroeste de la cabecera municipal de Dolores, Petén (Figura 1), en un paraje conocido como La Borrachera, en un terreno rodeado de colinas. Durante los siglos ocho y nueve $\mathrm{dC}$, y probablemente durante otros períodos también, Ixtutz era un centro importante para la dinastía Ho'kab, una de las familias de élite que reinaban en las montañas mayas occidentales (Mathews 1976; Zender 2002), y que probablemente tenía el sitio vecino de Ixtonton, en la meseta de Dolores, como su capital principal (Carter y Santini 2019a).

Ixtutz corresponde a la cuenca del río Poxte, por lo cual a nivel geográfico pertenece a la vertiente occidental de las montañas (Laporte y Escobedo 2009). El sitio está asociado a una zona pantanosa que hace difícil el acceso en épocas de invierno. El área central del sitio y algunos otros grupos habitacionales se encuentran protegidas por el Instituto de Antropología e Historia (IDAEH) y corresponden aproximadamente a 1 $\mathrm{km}^{2}$. La vegetación que predomina actualmente es primaria en algunos sectores del sitio, pero debido a los incendios forestales en esta área se ha ido reduciendo, tal cual fue registrado en el último incendio en abril de 2019, el cual consumió parte de la sección noroeste del sitio. El sitio solía estar rodeado de selva tropical con abundantes árboles de cacao y palmas de coroza
(Robertson 1972); ahora, en los alrededores predominan los potreros dedicados a la crianza de ganado vacuno.

Ixtutz fue una de las primeras ciudades Clásicas que se agregaron al plano arqueológico de Petén. Laporte y Escobedo (2009:46-47) documentaron claramente la línea de tiempo del sitio desde su descubrimiento por el coronel Modesto Méndez, Gobernador de Petén, que visitó Ixtutz y el sitio vecino Ixkun en 1852 (Ritter 1853). En la época moderna el sitio recibió atención renovada con visitas por exploradores como Merle Greene Robertson, en 1970, 1971 y 1972 (Robertson 1972), Ian Graham y Eric von Euw, en 1972 (Graham 1980:172) y Arlen y Diane Chase, en 1977 (Chase y Chase 1983:3). Robertson descubrió 15 bloques del Panel 1; luego, Graham y von Euw removieron 11 de ellos y la Estela 4 a Dolores, para su resguardo. Llevaron a cabo una serie de exploraciones menores en el sitio, dirigidas principalmente a mejorar el plano del área central mediante la definición de los rasgos arquitectónicos de las estructuras de la plaza principal. Se descubrieron 14 fragmentos del Panel 2 entre 1985 y 1989 por el Proyecto Nacional Tikal y el AAG; algunos quedan en la bodega del AAG y otros se exhiben en el Museo Regional del Sureste de Petén (Laporte 2009:117-118). El AAG ha conducido investigaciones en Ixtutz desde entonces hasta la actualidad.

En el plano actualizado por el AAG, el área central de Ixtutz se extendió a 10 plazas y 42 grupos con características habitacionales. En cuanto a la cronología del asentamiento, materiales cerámicos que se recuperaron de la superficie y de excavaciones en los pisos superiores de la Plaza A pertenecen a alguna parte de la fase Tepeu (véase Smith 1955), es decir, al período Clásico tardío y/o terminal (600-900 dC). Tiestos recuperados de niveles inferiores indican una ocupación posible de la fase Chicanel (Preclásico tardío, $400 \mathrm{aC}-250 \mathrm{dC}$ ) o la Chicanel Periférica (Clásico temprano, 250-600 dC), pero estos tiestos no están asociados con estructuras permanentes. Otros grupos arquitectónicos en el centro del sitio y en su periferia han sido explorados en excavaciones recientes conducidas gracias al apoyo de la Escuela de Historia y del Centro Universitario de Petén de la Universidad de San 


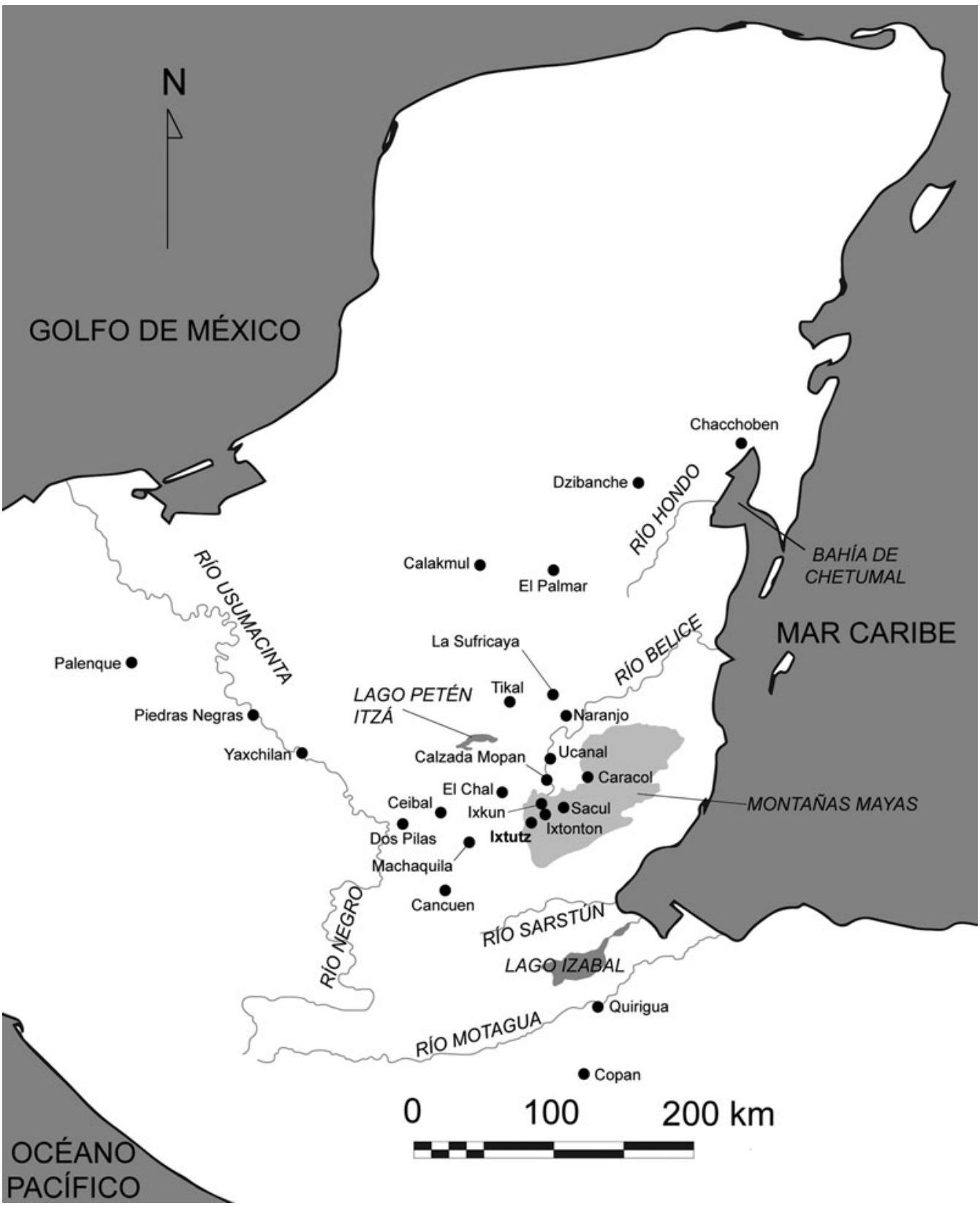

Figura 1. Mapa de la región maya por Nicholas Carter, usando contornos del Electronic Atlas of Maya Sites, (C) 2001, 2002, 2005, 2008, 2009, 2010 por Walter Witschey y Clifford T. Brown.

Carlos, dando resultados interesantes que han permitido redireccionar algunas cuestiones en el esquema político y cronológico de Ixtutz.

La Banca Jeroglífica 1 de Ixtutz consta de al menos cuatro bloques grabados, aquí designados como los Bloques 1, 2, 3 y p4. Es probable que originalmente existieran por lo menos seis. Los Bloques 1b, 2 y p4 fueron descubiertos por el AAG en el Grupo 17A, localizado sobre una serranía elevada al noroeste del área central del sitio (Figura 2). Este conjunto está conformado por seis estructuras localizadas sobre una 

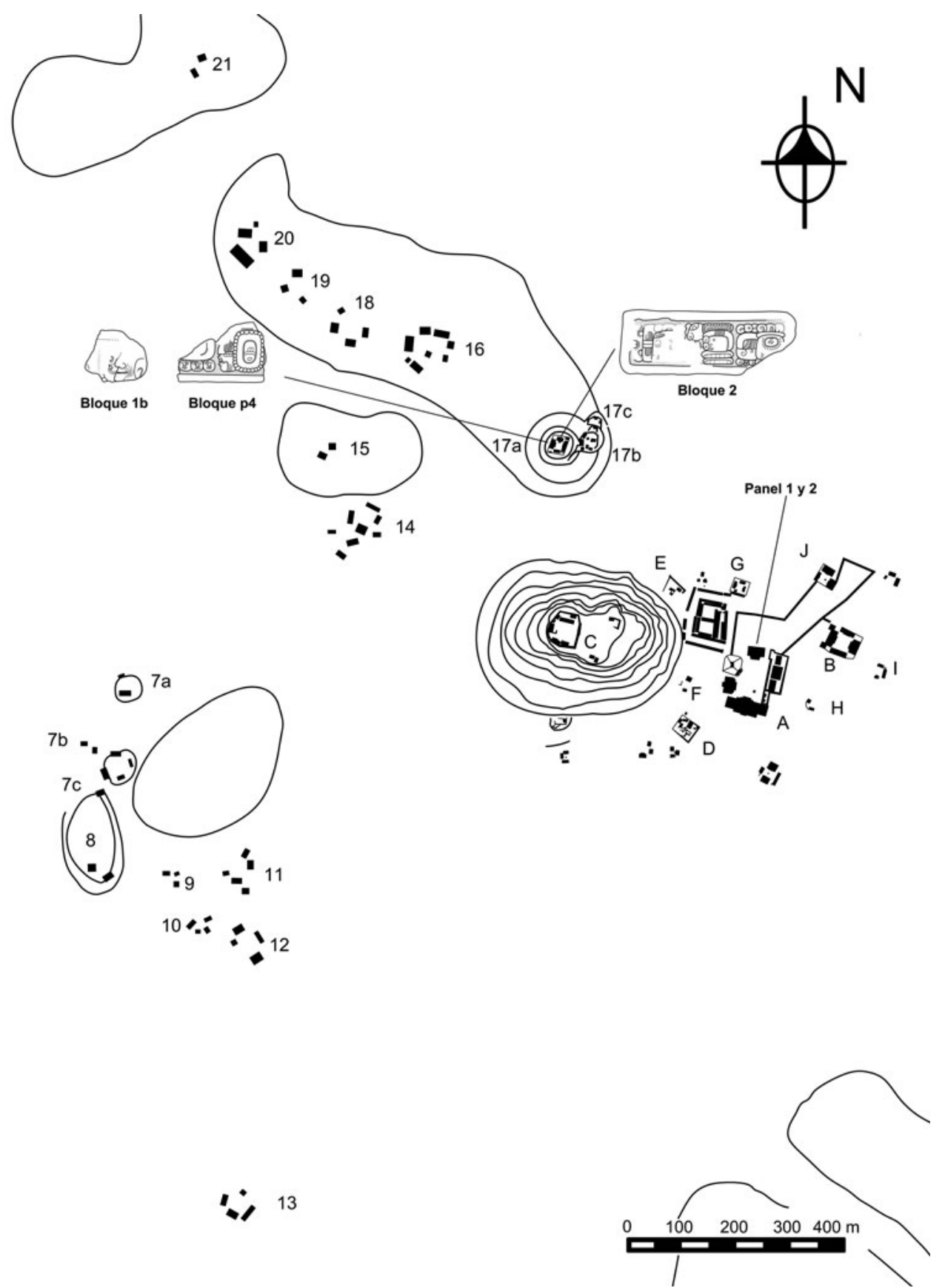

Figura 2. Mapa del centro de Ixtutz con las ubicaciones de los bloques de la Banca 1 y los Paneles 1 y 2, cortesía del Atlas Arqueológico de Guatemala.

plataforma, donde excavaciones recientes han definido dos etapas constructivas. La primera de ellas pertenece a la fase cerámica Tepeu I (600-700 dC), cuando fueron construidas las estructuras Este y Oeste; la segunda corresponde a la fase Tepeu II (700-800 dC), cuando la plataforma donde se sostiene el grupo fue ampliada, cubriéndose la primera plataforma con esquinas 
redondeadas, para permitir la construcción de las estructuras Norte, Sur, Noreste y Sureste. Tiestos cerámicos de las fases Chicanel y Chicanel Periférica también se recubrieron, pero sin asociación arquitectónica clara. Pequeñas cantidades de material Clásico terminal y Posclásico indican alguna actividad en el Grupo 17A durante esos períodos, pero no son suficientes para clarificar el grado de ocupación.

El Bloque 2 se encontró en junio de 2017, encima del piso de una pequeña plataforma frontal que dio acceso a la Estructura Norte del Grupo 17A, en una posición invertida (Figura 3). El Bloque $1 \mathrm{~b}$ fue encontrado durante exploraciones superficiales del tercer cuerpo de la Estructura Oeste en noviembre de 2017, y el Bloque p4 en el mismo contexto en junio de 2018. Por eso, sospechamos que el contexto original de la banca puede haber sido el interior de la Estructura Oeste.

\section{La reconstrucción virtual y una lectura de la Banca Jeroglífica 1}

Después del descubrimiento del Bloque 2, David Stuart sugirió que podría coincidir con un bloque inscrito en la Galerie Emile Deletaille, en Bruselas, que durante mucho tiempo se sospechaba que procedía de Ixtutz. El bloque de la colección Deletaille aquí se denomina el Bloque 3 de la banca. Trabajo de detective adicional por Stuart, Stephen Houston, Nicholas Carter y Megan O’Neil indicó que un bloque en la colección de LACMA, que en este trabajo designamos el
Bloque 1a, perteneció a la misma escultura (Figuras 4a y 4b). Estas sugerencias se basaron en múltiples líneas de evidencia. Las dimensiones de los bloques son casi las mismas: el Bloque 2 mide $15 \mathrm{~cm}$ de altura $\times 38 \mathrm{~cm}$ de ancho, y el Bloque 3, $15 \times 36 \mathrm{~cm}$ (Mayer 1978:14); el Bloque $1 \mathrm{a}$, que retiene aproximadamente $60 \%$ de su tamaño original, mide $14,732 \times 22,352 \mathrm{~cm}$ (Los Angeles County Museum of Art 2020).

También son muy parecidos el grosor de los marcos o bordes en cada bloque (aproximadamente $4 \mathrm{~cm}$ ), los espacios entre los marcos y los glifos (aproximadamente $1 \mathrm{~cm}$ ), y los tamaños de los glifos mismos (aproximadamente $8 \mathrm{~cm}$ de altura $\times 11 \mathrm{~cm}$ de ancho). Más evidencias incluyen el estilo caligráfico de la inscripción, la calidad redonda de los glifos y la profundidad del relieve. El descubrimiento in situ del Bloque $1 \mathrm{~b}$, que encaja con el Bloque 1a, confirmó la identificación.

Juntos, los Bloques 1a, 1b, 2 y 3 conforman un texto coherente que describe la dedicación de un trono. Es casi seguro que el Bloque p4 pertenece al mismo trono, o por lo menos al mismo programa monumental, considerando sus dimensiones, el estilo de los glifos y la presencia de la misma fecha que se encuentra en el Bloque 1a. Sin embargo, su relación sintáctica a los otros bloques no está clara; por eso lo llamamos el Bloque $\mathrm{p} 4$, donde $p$ significa provisional. Incluimos a continuación una transliteración, una transcripción y una traducción provisional del texto de los Bloques 1-3. Para los signos que no tienen desciframientos seguros, usamos las designaciones de

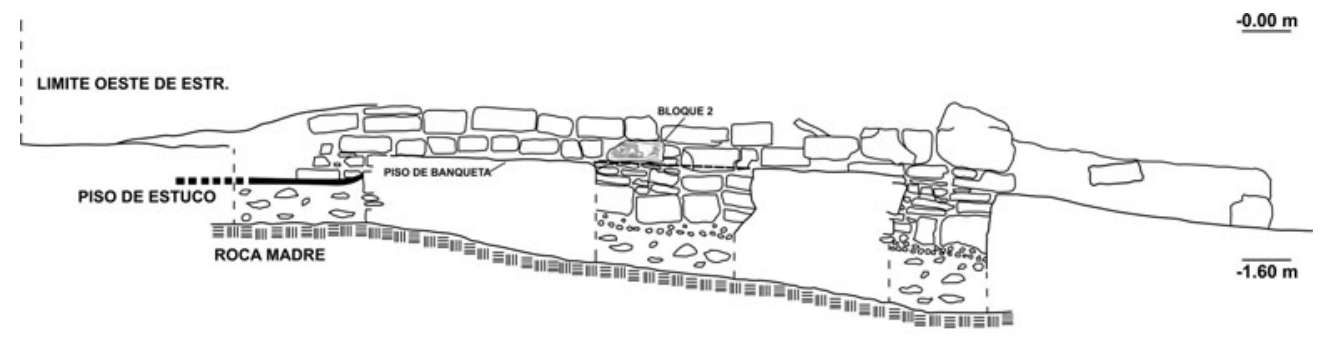

$\overline{-2.00 \mathrm{~m}}$

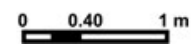

Figura 3. Perfil de excavaciones realizadas en la Estructura Norte del Grupo 17a con el Bloque 2 de la Banca 1, cortesía del Atlas Arqueológico de Guatemala. 
los catálogos jeroglíficos de Thompson (1962) y Macri y Looper (2003).

\author{
Bloque la \\ pA1 10-K'AN \\ lajuun k'an(?) \\ [En el día] $10 \mathrm{~K}$ 'an \\ pB1 12-mo-(lo) \\ lajchan mol \\ el 12 de Mol
}

\section{Bloque $1 b$ \\ pC1 T'AB-yi \\ t'abayi \\ se sube (i.e., "se dedica")}

\section{Bloque 2}

$\begin{array}{ll}\mathrm{pD} 1 & \text { U-ta-[?]-ji } \\ & u \text { ta[...]j(?) } \\ & \text { el } t a[\ldots] j(?) \text { de } \\ \mathrm{pE} 1 & \mathrm{U}-\mathrm{K} \text { 'UH-li-li } \\ & u \text { k'uhlil } \\ & \text { la cosa sagrada(?) de } \\ \mathrm{pF} 1 & \text { yu-ta-la-tz'a-(ma) } \\ & \text { yutal tz'am } \\ & \text { la cara del trono de }\end{array}$

Bloque 3

pG1 T505-AT3

[?]

[?]

$\begin{array}{cl}\text { pH1 } & \text { BAAK-T1013 } \\ & \text { baak [?] } \\ & \text { Hueso/Captivo [?] } \\ \text { pI1 K'AHK'-AJAW } & \text { k'ahk' ajaw } \\ & \text { señor [de] Fuego }\end{array}$

“[En el día] $10 \mathrm{~K}$ 'an $12 \mathrm{Mol}$ se dedica el $t a[$. . . lj de, la cosa sagrada(?) de la cara del trono de [. . .] Baak [. . .], el señor [de] Fuego."

Leídos en secuencia, los Bloques 1-3 describen la dedicación ( $t$ ' $a b)$ de un trono ( $t z^{\prime}$ 'am) que perteneció a un gobernante con el título real de K'ahk' ajaw, "Señor de [la corte] Fuego", en una fecha que se puede reconstruir como 9.18.13.16.4, $10 \mathrm{~K}$ 'an $12 \mathrm{Mol}$ (24 de junio, 804 dC, según la correlación 584,286 entre los calendarios maya y gregoriano; véase Martin y Skidmore 2012). Otras dos fechas son posibles:
9.16.1.3.4 (7 de julio, $752 \mathrm{dC}$ ) y 10.1.6.11.4 (11 de junio, $856 \mathrm{dC}$ ), pero preferimos 9.18.13.16.4 dada la probable identidad del rey que comisionó el trono (véase abajo).

Es notable que este texto contiene dos términos muy raros en el corpus maya - u-ta[...]-ji, en la posición D1, que es muy difícil de interpretar dado el daño al signo superior, y $u$ $k^{\prime} u h l i l$, en la posición E1. U k'uhlil es la forma poseída de un sustantivo k'uhlil que aparentemente se derive de k'uhul, "sagrado", a su vez derivado de k'uh, "dios", "cosa sagrada". Aunque $k$ 'uhlil es una expresión muy poco común —este puede ser el único ejemplo- es cognado con el término tzeltal ch'ulel, "alma", "suerte" (Laughlin 1988:200). En este contexto puede referir al alma viviente del trono o denotar el trono como un objecto sagrado. Un tercer término, yutal, es muy común en el corpus. Viene de una raíz ut, wut o hut, "cara", y por extensión "ojo", "fruta", "semilla" (Kaufman y Norman 1984:120; MacLeod 1990:391-395; véase Reents-Budet et al. 1994:161, nota 17). En casi todos los ejemplos, yutal aparece en los textos dedicatorios de vasijas cerámicas para cacao; por eso ha sido leído como "de frutas", en referencia a una bebida de cacao mezclada con fruta o preparada de la pulpa de vainas de cacao (Beliaev et al. 2010:258-260). El ejemplo de la Banca 1 es importante para la interpretación de tales textos porque en este caso, en referencia a un trono, una lectura como "de frutas" es muy improbable (véase Stuart 2006:188). Preferimos un sentido relacionado a "cara", tal vez en referencia a la superficie frontal o la cara del trono.

El nombre y el título del rey que comisionó el trono ocupan los tres bloques glíficos del texto, en Bloque 3. Sin entrar en demasiados detalles, su nombre es muy similar a los nombres de un rey de Ixtutz que fue responsable de por lo menos otros tres monumentos: la Estela 4 y los Paneles 1 y 2 , que de hecho son los únicos monumentos en Ixtutz con fechas jeroglíficas sobrevivientes, aunque las fechas de los paneles están incompletas (Laporte 2009:117; Figura 5). El nombre en el Bloque 3 comparte con el nombre real de la Estela 4 un componente que consiste en el logograma BAAK ("hueso" o "cautivo") y un signo no descifrado que representa la cabeza de un dios poco conocido, sin mandíbula. 
(a) Bloque 1a Bloque 1b
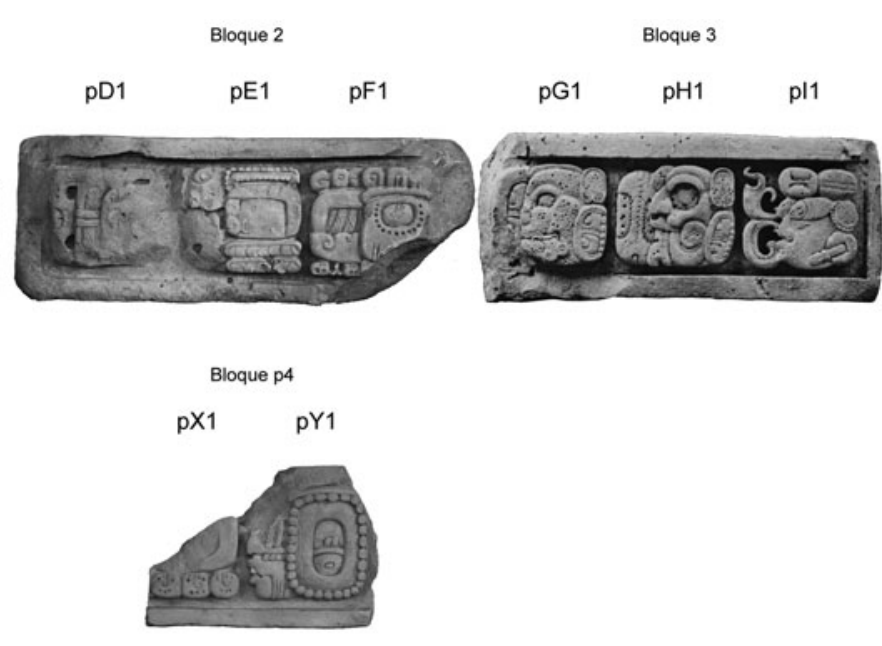

(b)
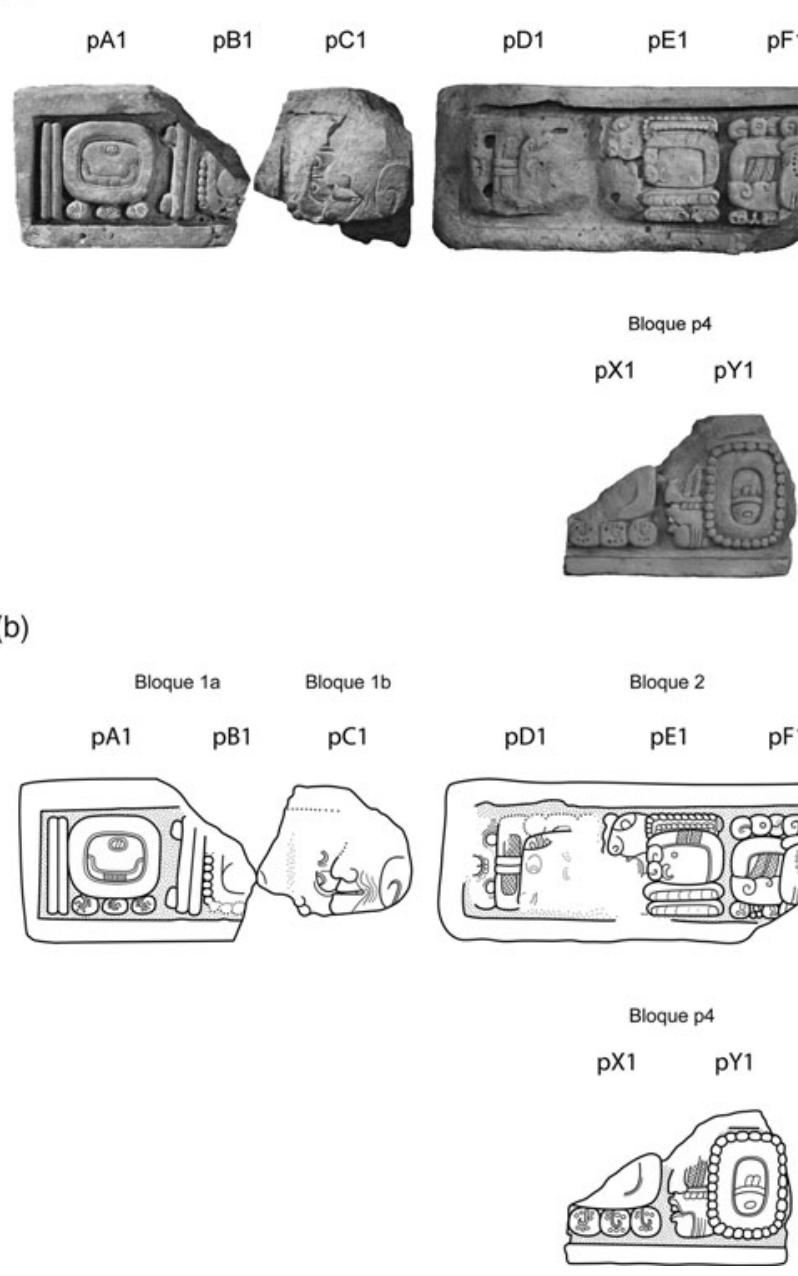

Figura 4. Reconstrucción del texto de la Banca 1 de Ixtutz. (a) Compuesto fotográfico; (b) dibujo por Nicholas Carter. La fotografía del Bloque 1a es propiedad de Justin Kerr, usada con permiso. La fotografía del Bloque 3 es por Ian Graham, regalo de Ian Graham, 2004. () President and Fellows of Harvard College, Peabody Museum of Archaeology and Ethnology, 2004.15.1.916.1.

Aunque los nombres no son completamente congruentes, sospechamos que pertenecen al mismo individuo, que llamamos "Gobernante 1" (en otros lugares conocido como "Aj Yaxjal"; véase Zender 2002).

Es importante tener en cuenta que el Bloque p4 duplica por lo menos la fecha haab (12 Mol) que se encuentra en el Bloque 1a, con una fecha en el calendario tzolk'in que no se puede leer, pero que es probablemente el mismo día 10 K'an del otro bloque. Si la fecha en el Rueda Calendárica es la misma o no, el texto fragmentario en el Bloque p4 no encaja con el resto de la inscripción conocida. No obstante, las proporciones del Bloque p4 y sus glifos, la profundidad de su relieve y el estilo de su escultura indican que es parte de la misma composición que los Bloques 1-3. La implicación clara es que existen otros bloques, ya sea no excavados o tal vez en colecciones privadas.

\section{Historia de un trono dividido}

Dadas las formas de los bloques y la referencia a un $t z$ 'am, "trono", es evidente que los bloques bajo consideración conformaron parte de un 


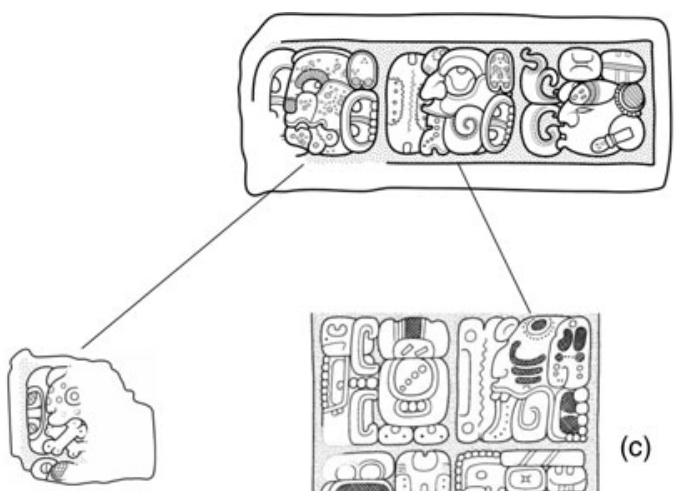

(a)

(b)

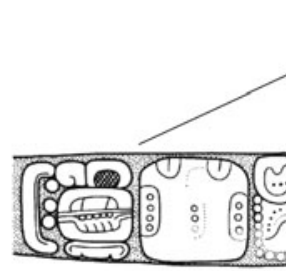

(d)

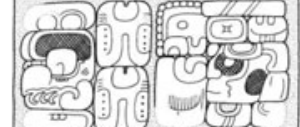

(c)

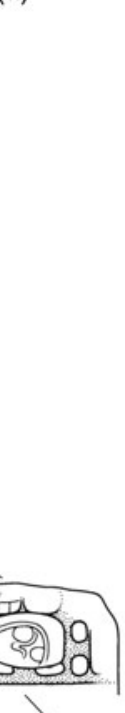

(e)
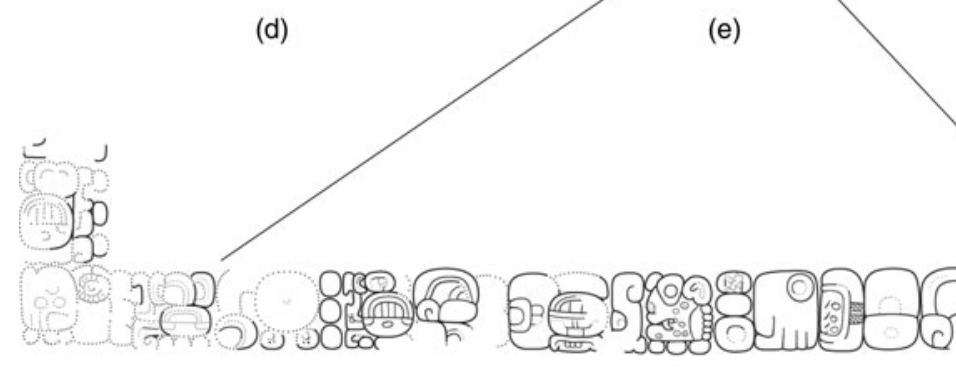

$\sqrt{6}$

(f)

Figura 5. Comparación de nombres y títulos reales de monumentos de Ixtutz, probablemente pertenecientes al Gobernante 1. (a) Banca 1, Bloque 3, bloques glíficos pG1-pI1 (T505-AT3 BAAK-T1013 K'AHK'-AJAW); (b) Fragmento Misceláneo E5, atribuido al Panel 2 (T505-AT3); (c) Estela 4 de Ixtutz, bloques glíficos A3-B4 (a-ya-YAX-ja-la BAAK-T1013 U-CHAN-na-bo-bo K'UHUL-lu-5-KAB-AJAW-wa); (d) Panel 1 de Ixtutz, Bloque XI, bloques glíficos 1-3 (U-CHAN-na bo K'UHUL-[. . . ]); (e) Panel 1 de Ixtutz, Bloque II, bloques glíficos 1-4 (WINIKHA'B pi-tzi-la K'UHUL-*5-KAB-AJAW-wa); (f) Panel 2 de Ixtutz, Bloques IV (bloques glíficos 1-6), V (bloques glíficos 1-6) y VI (bloques glíficos 1-4), espejados para legibilidad (3-wi-WINIKHA'B ch'a-ho-ma 3-wi-WINIKHA'B pi-PITZ-la 3-wi-WINIKHA'B ta-[. . . ] U-CHAN-na YAH-BAHLAM-ma ti-k'o jo-lo ta-ji K'UHUL-5-KAB-AJAW 8-WINIK-ki ba-ka-ba). Dibujos por Nicholas Carter; (c) y (f) según unas fotografías por el Atlas Epigráfico de Petén; (d) y (e) según unas fotografías por Ian Graham.

asiento real. Probablemente su forma era algo como la de la banca jeroglífica de la Estructura 9N-82 en Copan, la famosa "Casa de los Bacabes" (Webster 1989:30-34). El asiento de esa banca consiste en cinco losas de toba, sostenidas por una plataforma de unos bloques estrechamente colocados. Pero el borde inscrito de la banca se proyecta hacia adelante en relación con la mayor parte de la plataforma. Sólo seis soportes esculpidos están alineados con la parte delantera del asiento, simulando la apariencia de una banca con patas independientes (Figura 6; véase Webster 1989:Figura 14).

Tal composición colocaría la Banca Jeroglífica 1 de Ixtutz en una larga tradición de bancas ceremoniales en la región maya y en el mundo mesoamericano más amplio. La forma ancestral del trono real maya remonta a la cultura olmeca 


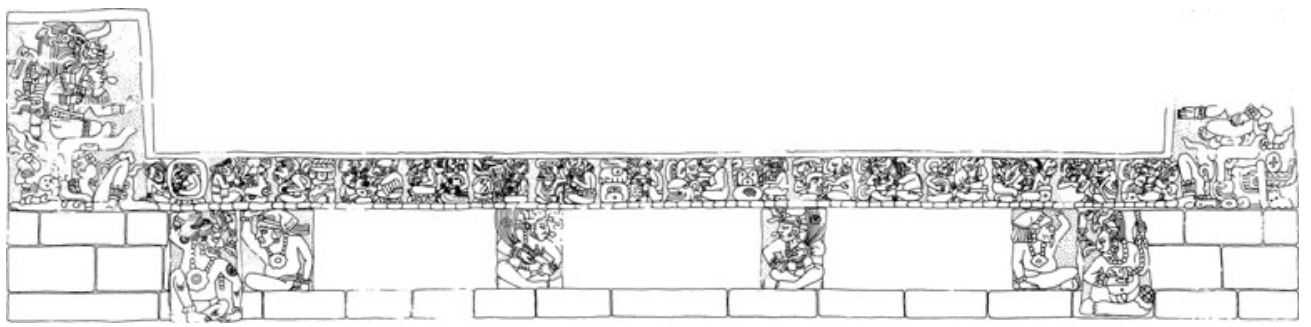

Figura 6. La banca jeroglífica del Cuarto 1 de la Casa de los Bacabes, Grupo 9N-82, Patio A, Copan. Dibujo por Anne Dowd, (c) David Webster, usado con permiso.

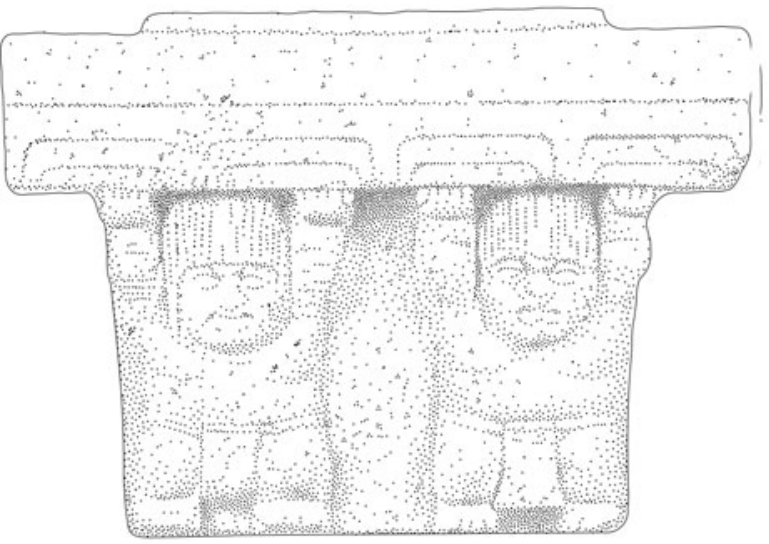

(a)

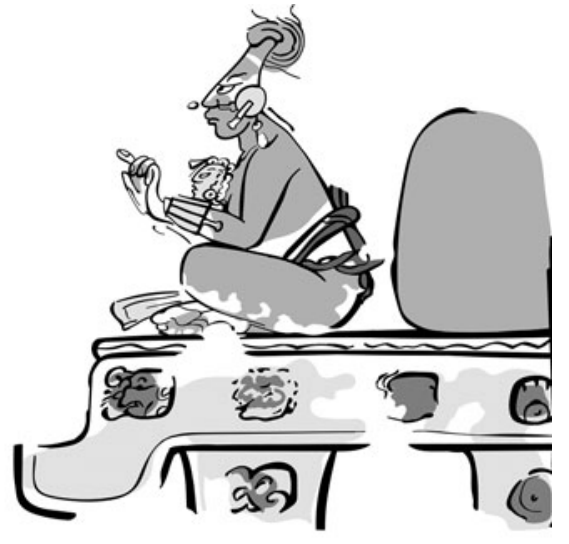

(b)

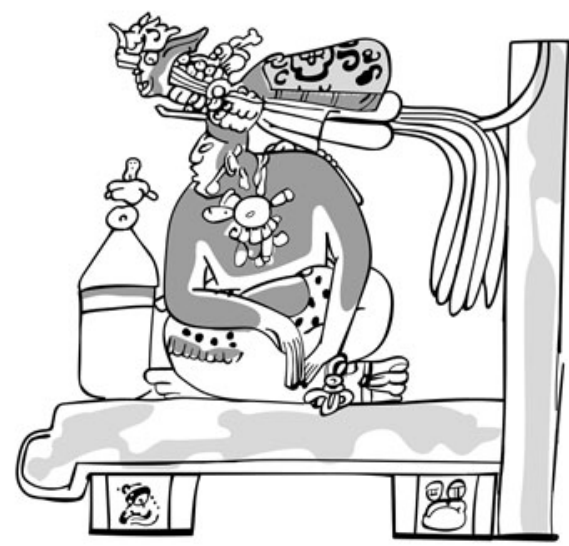

(c)

Figura 7. (a) Trono olmeca de piedra del período Formativo medio (Monumento 2 de Potrero Nuevo, Veracruz); (b) y (c) detalles de vasijas mayas del Clásico tardío, mostrando personajes sentados en bancas similares con textos jeroglíficos. Dibujos por Nicholas Carter: (a) según una fotografía por el Museo de Antropología de Xalapa; (b) y (c) según las fotografías K1524 y K2800 por Justin Kerr.

del Preclásico medio (aproximadamente 950$400 \mathrm{aC}$ ), de la que contamos con unos ejemplos de piedra (Figura 7a). Tronos similares aparecen en escenas pintadas en vasijas cerámicas mayas del Clásico tardío, donde están representados cubiertos con estuco o pintados, a veces con textos jeroglíficos (e.g., Coe 1973:68; Reents-Budet et al. 1994:222; Figuras 7b y 7c). Muchos de 
tales objetos probablemente fueron hechos de madera, pero algunas bancas mayas de la misma forma son de piedra, como la del Grupo 10K-4 en Copan (Baudez 1989:Figura 69).

Se puede hacer una comparación informativa entre la Banca 1 de Ixtutz y una presunta banca jeroglífica asociada con la Estructura 4 de Machaquilá, reconstruida virtualmente por Lacadena García-Gallo y fechada por él a la mitad del siglo nueve (Lacadena García-Gallo 2010:224226). En su reconstrucción, el asiento del trono consiste en diferentes bloques rectangulares de caliza, inscritos en sus caras con un texto continuo, con tres soportes también compuestos de múltiples bloques inscritos (Figura 8; Lacadena García-Gallo 2010:Figura 13). Como la Banca 1 de Ixtutz, los bloques de la banca de Machaquilá se encontraron desordenados y removidos de su contexto original, pero dado el número de distintos sillares es probable que requirieron un núcleo sólido de bloques para sostenerlos, como la banca de la Casa de los Bacabes. Los bloques del asiento de Machaquilá son muy similares a los de la Banca 1 de Ixtutz — sólo que el texto no está dividido por marcos en los extremos izquierdo y derecho de cada bloque, y que el estilo caligráfico de los glifos es algo diferente. Es evidente que las dos bancas pertenecen a un estilo o tradición regional, con escultores en diálogo uno con otro, respondiendo a las obras de los demás. Dado la mala condición de la mayoría de los monumentos de Ixtutz e Ixtonton, no es posible establecer fuertes vínculos paleográficos o sintácticos entre las inscripciones del reino Ho'kab y las de Machaquilá. Sin embargo, proyectos reales de Machaquilá sí participan en tradiciones estéticas ampliamente compartidas en la región maya del sureste, incluida la región de
Dolores y sitios como Caracol y Ucanal, como el uso de mampostería enchapada en edificios y la colocación de textos jeroglíficos en cartuchos redondeados en monumentos.

Si estamos en lo cierto acerca de su identidad, el Gobernante 1, el dueño de la Banca 1, probablemente fue responsable de gran parte del arte monumental y la construcción de la plaza principal (Zender 2002:19). El descubrimiento de los fragmentos de la Banca 1 en el Grupo 17A sugiere que él dejó su marca en otros sectores del sitio también. Pero ¿qué motivó la construcción del trono? 9.18.13.16.4 no es una estación importante en la Cuenta Larga ni, por lo que sabemos, un aniversario de cualquier evento importante en la historia del reinado Ho'kab. Pero sí corresponde al 24 de junio, entre días del solsticio de verano. Los solsticios tenían una evidente importancia en varios sitios mayas, donde se conmemoraron con bailes rituales y construcciones arquitectónicas (Dowd 2015; Tate 2013). El solsticio de verano muy bien puede haber proporcionado una razón religiosa para la construcción de la Banca 1.

También podemos sugerir razones prácticas y políticas para la dedicación de la banca como parte de un programa más amplio de construcción monumental en Ixtutz. Durante los fines del siglo siete y la primera parte del ocho, el reino Ho'kab y el reino vecino de Juluup, con su capital en el sitio de Sacul 1, eran partes de una red de alianzas y relaciones diplomáticas amistosas, más o menos fuertes, entre muchas entidades políticas mayas con los reyes Kaanal de Calakmul a la cabeza (Carter 2016; MacLeod 2022; Martin y Grube 2008:108-113). En Petén oriental, el sitio poderoso de Naranjo era un nodo clave en esa red desde la refundación de

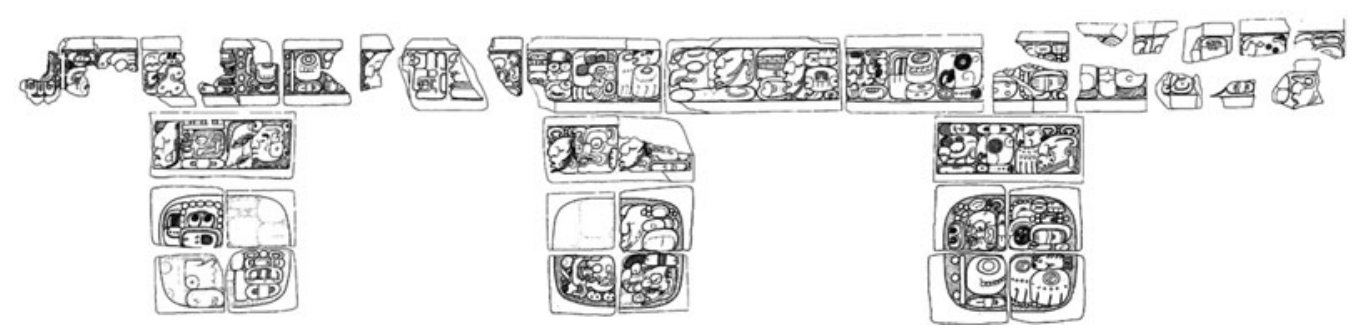

Figura 8. Reconstrucción de una banca jeroglífica de Machaquilá. Dibujo por Alfonso Lacadena García-Gallo, cortesía de Andrés Ciudad Ruiz. 
su dinastía bajo la supervisión de Kaanal alrededor del $680 \mathrm{dC}$ hasta su derrota por Tikal, el gran enemigo del reinado Kaanal, en 744 (Martin y Grube 2008:72-77).

La caída de Naranjo inició un período de desintegración entre las entidades políticas de las montañas mayas suroccidentales y las áreas adyacentes que habían formado parte de la alianza. Al principio, el rey de K'anwitznal, en el sitio de Ucanal al norte de la meseta de Dolores a lo largo del río Mopán, mantuvo unido un remanente de la red, con el gobernante de Juluup como uno de sus vasallos. Luego, el rey de Juluup instaló su propio vasallo, con Ixkun como su nueva capital, interrumpiendo la ruta natural para el comercio y los viajes que conectó Ixtonton y Ucanal a las regiones al norte y sur (Carter et al. 2019:Figura 12). Esta decisión precipitó una guerra entre K'anwitznal y la alianza Juluup-Ixkun en 779 y 780 dC (Carter 2016:244-247). Menos que un mes después de la victoria de Juluup sobre K'anwitznal, el rey de Juluup atacó otro sitio y tomó un cautivo. El glifo emblema del prisionero, en la Estela 6 de Sacul (tallada poco después del 7 de marzo, 780), parece ser lo de Ho'kab (Carter y Santini 2019b), sugiriendo que la corte Ho'kab se puso al lado de K'anwitznal en el conflicto. La evidencia para relaciones hostiles continuas entre Ho'kab y la alianza Juluup-Ixkun viene de la Estela 1 de Ixkun (790 dC), que representa un cautivo con el mismo título de $K^{\prime} a h k^{\prime}$ ajaw que leemos en la Banca 1.

En este contexto histórico, el sitio de Ixtonton, que tuvo acceso a las tierras relativamente planas y fértiles de la meseta de Dolores, y que estaba ubicado en una ruta natural por las montañas, puede haberse vuelto demasiado peligroso para usar como la capital principal de Ho'kab. Bajo amenaza de Juluup e Ixkun, el Gobernante 1 puede haber movido su sede real de Ixtonton a Ixtutz y empezado una campaña de construcción monumental que se extiende por lo menos desde la Estela 4 (780 dC) hasta la Banca 1 (804 dC). Después de su dedicación, se presume que la banca fue utilizada para audiencias o banquetes reales, un tema representado con frecuencia en escenas de los interiores de palacios pintadas en vasijas polícromas del Clásico tardío. En la vida cortesana de los mayas, las distinciones de elevación y postura — ¿quién se sentó? ¿en el piso o en una superficie elevada? y ¿quién estuvo de pie? ¿en qué lugar relativo al rey?- eran esenciales para la organización social del corte (Houston et al. 2006:174-175). Como tal, el desmantelamiento y reutilización de una banca real marcaría un cambio radical en la organización de la autoridad y las relaciones políticas entre una corte, aún el fin de la realeza misma.

Para la Banca 1 de Ixtutz, ese momento de desmontaje y recontextualización vino en algún momento del período Clásico terminal (830$900 \mathrm{dC}$ ) o en los principios del Posclásico temprano (900-1200). En toda parte de las tierras bajas mayas del sur, el Clásico terminal vio el colapso de las dinastías reales, el reemplazo de la realeza por otras formas de autoridad política y social, y la destrucción o reutilización de muchos monumentos reales - todo en el contexto de sequía generalizada, despoblación y la reconfiguración de rutas comerciales (e.g., Aimers 2007; Beach et al. 2016; Dunning et al. 2012). Pero esos cambios no sucedieron en el mismo momento en cada sitio. En la región de Dolores, las dinastías de Juluup e Ixkun desaparecieron alrededor del cambio del siglo nueve $\mathrm{dC}$, o al menos antes del fin del bak'tun 10.0.0.0.0 en 830. El último monumento en Sacul 1 con una fecha sobreviviente es la Estela 9, dedicada en 790, y el último en Ixkun es la Estela 5, en 800 (Laporte y Mejía 2005:160). Los monumentos de ninguno de los dos sitios muestran signos de mutilación en la antigüedad. Sin embargo, la familia real de Ixkun puede haber tenido un final espantoso: los esqueletos de por lo menos 13 personas, de diferente edad y sexo, con adornos y modificaciones dentales, encontrados por el AAG amurallados en una cueva en la colina sagrada en el extremo sur del sitio, pueden pertenecer a miembros de la casa real que fueron sellados vivos en la cueva (Mara Antonieta Reyes a Nicholas Carter, comunicación personal 2019). En Sacul 1, por lo menos, los grupos centrales arquitectónicos del sitio fueron ocupados en el Posclásico por una población plebeya pequeña (Laporte y Mejía 2006).

En el reinado Ho'kab, por otra parte, el siglo nueve fue un período de poder y prosperidad en comparación a los otros centros de población 
anteriores de la región de Dolores, aun cuando los tiempos no eran tan buenos como en el apogeo de la alianza Kaanal. La evidencia cerámica sugiere una ocupación reducida para Ixtutz, sin nuevas construcciones monumentales (Laporte 1992:419); con la amenaza de Juluup e Ixkun removida, el centro de gravedad de la dinastía puede haber regresado a Ixtonton. Ese sitio experimentó construcción mayor, incluido el crecimiento y la densificación de los edificios residenciales (Laporte y Mejía 2002:71, 82; Laporte y Quezada 1998:852). La arquitectura monumental de Ixtonton en el siglo nueve muestra una fuerte influencia del norte de la península Yucatán, como la presencia de mascarones de mosaico que representan el dios de la lluvia Chahk en la última fase de la Pirámide Sur de la Plaza A de la Acrópolis. Mientras tanto, en la Plaza B del mismo grupo hay evidencia de ocupación en el edificio palaciego y sobre un edificio ya colapsado (presumiblemente en estructuras perecederas). Esta ocupación Clásica terminal incluyó la presencia de unos fragmentos de monumentos removidos del Grupo Central del sitio (Laporte y Quezada 1998:853). A pesar del hecho que estos monumentos se habían quebrado, es evidente que la dinastía Ho'kab continuó en poder en Ixtonton hasta el fin del siglo nueve, porque un rey de esa línea dedicó la Estela 1 de Ixtonton para el fin del k'atun 10.3.0.0.0 en 889 — probablemente mucho después de la reubicación de los fragmentos del Grupo Central.

En muchos casos, como en Copan (Andrews y Fash 1992; Maca 2002), El Zotz (Newman et al. 2018), Ucanal (Halperin y Garrido 2020) y El Perú-Waka' (Eppich 2012:312), el fin de la realeza y la terminación de monumentos y edificios reales no significaron ni el abandono de un sitio ni el fin de jerarquía social, y los habitantes restantes de un sitio reutilizaron partes de monumentos reales para nuevos propósitos arquitectónicos, a veces para construcciones con un valor público o ritual. En tales instancias, puede ser difícil distinguir un amplio rango de potenciales tipos de significado que podrían ser inherentes a la reutilización de un monumento anteriormente venerado. Éstos pueden incluir la reutilización oportunista de piedras talladas que habían perdido su significado social, la terminación respetuosa de un objecto valorado, la profanación de los monumentos de un enemigo o un régimen recientemente pasado de moda, la curación y veneración de un objeto tal vez dañado contra la voluntad de los cuidadores, o un deseo general de asociarse con un pasado tal vez comprendido vagamente.

A nuestros ojos, el desmantelamiento de la Banca 1 de Ixtutz aparece como un acto de profanación y falta de respecto. El Bloque 2 fue removido de su contexto original, puesto al revés y reutilizado como grado de acceso. Varios otros bloques del trono se sacaron de la Estructura Oeste y se descartaron en la plataforma del edificio. El Bloque 1 puede que se haya roto durante ese evento. Aunque sus contextos de descubrimiento están perdidos en el tiempo, la falta de excavaciones de saqueo obvias en Ixtutz sugiere que los Bloques 1a y 3 se encontraron en contextos superficiales, muy parecidos a los Bloques $1 \mathrm{~b}$ y $\mathrm{p} 4$. Por lo tanto, contamos con una situación en que un bloque del trono real fue reutilizado en una manera insultante, mientras que los otros fueron dispersados fuera de su antigua casa. Esta situación nos aparece algo diferente a la de la Plaza B de la Acrópolis de Ixtonton, donde se curaron fragmentos de monumentos del Grupo Central. Por eso, sugerimos que la Banca 1 fue desmantelada después del fin de la dinastía Ho'kab — es decir, después de 889 dC - mientras que la reubicación de monumentos en Ixtonton puede representar el rescate más temprano de reliquias valoradas por una dinastía Ho'kab resurgente.

Restos cerámicos muy limitados sugieren que la ocupación de Ixtutz durante el período Posclásico temprano era pequeña, tal vez esporádica. Ixtonton continuó poblado durante el Posclásico, pero para el momento en que Hernán Cortés y sus soldados pasaron por las montañas mayas en 1525, probablemente por medio de la meseta de Dolores, no encontraron ningún asentamiento, y sus guías les informaron que la región era completamente despoblada (Cortés 1868:6061). El pueblo de Dolores fue establecido en 1708 por el maestro de campo Juan Antonio Bustamante, y aunque se puede presumir que Ixtutz era conocido por algunos de sus ciudadanos, la existencia del sitio no fue publicada hasta que el coronel Modesto Méndez y Eusebio Lara 
visitaron en 1852 (Ritter 1853). Cuando un residente de Dolores, Mirando Huil Obando, condujo a Merle Greene Robertson a las ruinas de Ixtutz en julio de 1970, él indicó que su familia había conocido el sitio durante muchos años, pero que pensó que nadie más lo había visitado (Robertson 1972:89).

La ubicación de Ixtutz se hizo de conocimiento común muy pronto, cuando Robertson, Graham y von Euw pasaron tiempo en el sitio durante los próximos dos años, acompañados por trabajadores de Dolores, y publicaron unos relatos de sus exploraciones y descubrimientos (Graham 1980; Robertson 1972). Para ese tiempo, esculturas mayas antiguas habían adquirido un nuevo significado como obras de "arte primitivo" con valor monetario en el mercado internacional de arte (Coggins 1972). Unas décadas de trabajo arqueológico en la región maya habían llevado las antigüedades mesoamericanas a la atención del público global y del mundo de los comerciantes y conocedores de arte, creando una demanda fuerte de tales obras. La demanda de mercado condujo directamente a un epidémico de depredación de muchos sitios arqueológicos y la exportación ilegal de monumentos, vasijas y otras antigüedades a gran escala. En esta situación, Graham y von Euw percibieron tanta amenaza para los monumentos de Ixtutz en 1972 que decidieron en trasladar los mejor conservados de los que conocían — es decir, la Estela 4 y los bloques conocidos del Panel 1a Dolores para su seguridad. Y sus miedos no eran infundados, porque fue alrededor de este tiempo que alguien descubrió y quitó los Bloques 1a y 3 de la Banca 1 de Ixtutz.

No se sabe exactamente la fecha cuando los bloques fueron saqueados de Ixtutz. Sus viajes posteriores también son oscuros, al igual que los canales por los que llegaron a sus próximos destinos. No obstante, ninguno de los bloques salió a luz antes de mediados de la década de 1970. No hay evidencia de que ninguno de ellos haya sido descubierto o sacado de Guatemala antes de esa fecha. Este punto es importante porque el Decreto No. 425 de 1947 prohibió la exportación de objectos arqueológicos, definidos como "todas las estructuras y restos o vestigios procedentes de las civilizaciones aborígenes anteriores a la conquista", excepto para propósitos de exhibición pública e investigación científica, sujeto al permiso del estado guatemalteco. Así, a menos que los Bloques 1a y 3 fueran removidos de Guatemala antes de 1947 —una posibilidad muy improbable, y por lo que sabemos completamente sin evidencia documentaria-su exportación fue ilegal.

El Bloque 3 estaba en una colección privada en Bruselas, la Galerie Emile Deletaille, a más tardar en 1976, el año de la exposición "Art de Mesoamerique" en esa ciudad y la publicación del catálogo epónimo (Van Swieten 1976:no. 163). Ya se infirió que el bloque probablemente vino de Ixtutz dado el nombre compartido con la Estela 4 de ese sitio, pero también se consideró la posibilidad de que viniera de algún centro vecino como Ixkun o Sacul (Escobedo 1993; Mayer 1978:14-15). El Bloque 3 permanece en la colección Deletaille hasta el día de hoy.

El Bloque 1a fue adquirido por LACMA en 2010 de la colección del banquero Lewis Ranieri, que a su vez lo había obtenido del Fine Arts Museum of Long Island (FAMLI). Fundado en 1978, FAMLI sufrió problemas financieros durante la década de 1990 y cerró permanentemente en 1997, pero la dispersión de sus colecciones fue retardada por procesos legales hasta 2003 (Mead 2003). Dado el saqueo ilícito del bloque y la consecuente pérdida de toda información contextual, hasta la reconstrucción presentada aquí se podría haber dicho muy poco sobre el Bloque 1a. El sitio web de LACMA lo describe como un "fragmento de un panel o una banca tallada", atribuida a Guatemala o México, desde 600 hasta 900 dC (Los Angeles County Museum of Art 2020).

\section{Conclusión}

En este ensayo hemos mostrado que dos esculturas mayas del siglo nueve, anteriormente no reconocidos como partes de la misma obra, pertenecen a un solo trono real, la Banca Jeroglífica 1 del sitio de Ixtutz, Guatemala, un centro real de la dinastía Ho'kab. Hemos presentado los fragmentos de la banca descubiertos in situ por el Atlas Arqueológico de Guatemala, y sospechamos que aún existen otros bloques por descubrir. Fechamos la banca al 804 dC, y tentativamente la atribuimos al mismo rey —el Gobernante 1- 
que comisionó todos los otros monumentos legibles de Ixtutz, la Estela 4 y los Paneles 1 y 2, en las secuelas de un conflicto entre el reinado Ho'kab y las cortes vecinas de Juluup e Ixkun.

El texto de la Banca 1 presenta unos datos epigráficos nuevos e interesantes, como la apariencia del término k'uhlil, el empleo de yutal en un contexto donde tiene que significar algo como "su cara" en lugar de "de frutas", y una confirmación de que $K$ 'ahk' ajaw ("Señor de Fuego") era un título real de los reyes de Ho'kab. Pero tal vez se debe considerar el valor de la banca menos en términos epigráficos y más en términos de otros tipos de significación. Los significados de la banca cambiaron con el tiempo: construida para ceremonias y audiencias cortesanas temprano en el siglo nueve, la Banca 1 fue desmantelada después de $889 \mathrm{dC}$, sus bloques descartados o reutilizados en una manera que revela una notoria falta de respeto para la (presumiblemente difunta) dinastía Ho'kab. Olvidados durante muchos siglos, dos fragmentos del trono adquirieron un nuevo significado como objetos de arte durante un período cuando las obras de las culturas indígenas de Mesoamérica, África, Oceanía y el sudeste de Asia tenían un rango de valores nuevamente salientes: un valor monetario, por supuesto, pero también como evidencia sobre culturas pasadas, símbolos de identidades nacionales y como inspiración para artistas modernos. Fue en esta capacidad que los Bloques 1a y 3 atrajeron a coleccionistas, escolares y audiencias de exhibiciones de museos.

Con el descubrimiento de los Bloques 1b, 2 y p4, y la recontextualización de los bloques en Los Ángeles y Bruselas, la Banca 1 forzosamente adquiere nuevos significados. Por primera vez se puede leer y derivar información histórica y lingüística de una sección contigua del texto de la banca que se extiende por múltiples bloques. Los hallazgos arqueológicos nos permiten rastrear la banca a un edificio, en un sitio, en un año - un grado de especificidad no posible hasta ahora. Este conocimiento crea nuevas oportunidades y responsabilidades para cooperación entre países, entre el mundo de galerías y museos y el de la arqueología. Es raro que se presente una oportunidad de este tipo, y todas las partes interesadas deben actuar rápidamente para repatriar este fragmento del patrimonio enajenado de Guatemala.
Se pueden señalar varios ejemplos de trabajo de detective arqueológico que resultó en la repatriación de artefactos del mundo maya. Tales esfuerzos eran un componente importante del trabajo del Corpus of Maya Hieroglyphic Inscriptions según lo diseñado por su fundador Ian Graham. Un buen ejemplo es el caso del Dintel 1 de El Zotz, Petén, Guatemala. Como los bloques de Ixtutz, este dintel de chicozapote apareció en el mercado de arte en la década de 1970, entró una colección privada y luego un museo público — en este caso, el Denver Art Museum en Colorado. El trabajo de Graham mostró que el dintel en Denver emparejó a unos fragmentos dejados in situ en una estructura en El Zotz. En 1998, el museo regresó el Dintel 1 a Guatemala en un acuerdo voluntario; ahora el dintel queda reunido en exhibición en el Museo Nacional de Arqueología y Etnología (Houston 2008:6).

Tales historias exitosas son posibles sólo cuando el sitio de donde vino un artefacto saqueado ha sido identificado, permitiendo al gobierno pertinente presentar un reclamo. El peligro en tales situaciones es que la identificación pueda aumentar el precio de un artefacto robado. En este caso, consideramos que, como con el dintel de El Zotz, la Republica de Guatemala tiene un reclamo legal y moral a las piezas de la Banca 1 de Ixtutz que fueron exportadas ilegalmente y que permanecen en el extranjero hoy. Por primera vez es posible demonstrar este reclamo conclusivamente; es esta certeza que, en nuestra opinión, justifica la publicación de información que, sin duda, tiene el efecto incidental de validar la autenticidad de dos artefactos que hasta ahora habían estado sin procedencia. Tenemos razones para creer que los beneficios de esta información a la arqueología y sobre todo a la recuperación del patrimonio cultural de Guatemala pesan más que el riesgo de afectar las valoraciones de mercado de los Bloques 1a y 3. A pesar de su exportación ilegal, los actuales propietarios de los bloques en Bruselas y Los Ángeles han sido buenos guardianes de ellos - pero es hora de traerlos a casa. En el caso del bloque en Los Ángeles, parece probable un final feliz: el Programa del Arte de las Américas Antiguas está en proceso de preparar una propuesta para la junta de LACMA para repatriar el bloque a Guatemala (Diana Magaloni a 
Nicholas Carter, comunicación personal 2021). Alabamos a LACMA por dar este paso, y pedimos a la Galerie Deletaille que inicie conversaciones con el gobierno guatemalteco sobre la repatriación del bloque en Bruselas también, para que todas las partes de la Banca 1 puedan reunirse y exhibirse juntos en un museo en su país de origen.

Agradecimientos. Agradecemos la ayuda indispensable de Alyce de Carteret, Andrés Ciudad Ruiz, Barbara Fash, Cynthia Mackey, Diana Magaloni, Karl Herbert Mayer, Mary Miller, F. Kent Reilly III, Alexandre Tokovinine, David Webster, Donna Yates y tres revisores anónimos. También deseamos agradecer a Julia Hendon por animarnos a defender éticamente la publicación y repatriación de los bloques de la Banca Jeroglífica 1, y por presentar nuestro caso al Publication Committee de la SAA. Como el Atlas Arqueológico de Guatemala es un departamento de la Dirección General del Patrimonio Cultural y Natural de Guatemala, no se requirieron permisos para realizar sus excavaciones en Ixtutz. Los autores no tienen conflictos de intereses económicos o de otro tipo.

Declaración de disponibilidad.. Los Bloques 1b, 2 y p4 de la Banca Jeroglífica 1 de Ixtutz están en el laboratorio del Atlas Arqueológico de Guatemala en Dolores, Petén.

\section{Referencias citadas}

Aimers, James J.

2007 What Maya Collapse? Terminal Classic Variation in the Maya Lowlands. Journal of Archaeological Research 15:329-377.

Andrews, E. Wyllys y William L. Fash

1992 Continuity and Change in a Royal Maya Residential Complex at Copan. Ancient Mesoamerica 3:63-88.

Baudez, Claude

1989 The House of the Bacabs: An Iconographic Analysis. En The House of the Bacabs, Copan, Honduras, editado por David Webster, pp. 73-81. Studies in Pre-Columbian Art and Archaeology 29. Dumbarton Oaks, Washington, DC.

Beach, Timothy, Sheryl Luzzadder-Beach, Nicholas Dunning y Duncan Cook

2016 Climatic Changes and Collapses in Maya History. Past Global Changes Magazine 24(2):66-67.

Beliaev, Dmitri, Albert Davletshin y Alexandre Tokovinine

2010 Sweet Cacao and Sour Atole: Mixed Drinks on Classic Maya Ceramic Vases. En Pre-Columbian Foodways: Interdisciplinary Approaches to Food, Culture, and Markets in Ancient Mesoamerica, editado por John E. Staller y Michael D. Carrasco, pp. 257-272. Springer, New York.

Carter, Nicholas P.

2016 These Are Our Mountains Now: Statecraft and the Foundation of a Late Classic Maya Royal Court. Ancient Mesoamerica 27:233-253.

Carter, Nicholas P. y Lauren Santini

2019a Epigraphy and the Archaeology of Settlement in the Dolores Region, Peten, Guatemala. Ponencia presentada en la $84^{\mathrm{a}}$ Reunión Anual de la Society for American Archaeology, Albuquerque, New Mexico.

2019b The Lord of Yellow Tree: A New Reference to a Minor Polity on Sacul Stela 9. PARI Journal 19(4):1-9.

Carter, Nicholas P., Lauren M. Santini, Adam Barnes, Rachel Opitz, Devin White, Kristin Safi, Bryce Davenport, Clifford Brown y Walter Witschey

2019 Country Roads: Travel, Visibility, and Late Classic Settlement in the Southern Maya Mountains. Journal of Field Archaeology 44:82-104. DOI:10.1080/ 00934690.2019 .1571373$.

Chase, Arlen F. y Diane Z. Chase

1983 Intensive Gardening among the Late Classic Maya. Expedition 25(3):2-11.

Coggins, Clemency

1972 Archaeology and the Art Market. Science 175:263266.

Coe, Michael D.

1973 The Maya Scribe and His World. Grolier Club, New York.

Cortés, Hernán

1868 The Fifth Letter of Hernan Cortes to the Emperor Charles V, Containing an Account of His Expedition to Honduras. Traducido y editado por Don Pascual de Gayngos. Hakluyt Society, London.

Dowd, Anne S.

2015 Maya Architectural Hierophanies. En Cosmology, Calendars, and Horizon-Based Astronomy in Ancient Mesoamerica, editado por Anne S. Dowd y Susan Milbrath, pp. 37-75. University Press of Colorado, Boulder.

Dunning, Nicholas, Timothy Beach y Sheryl Luzzadder-Beach 2012 Kax and Kol: Collapse and Resilience in Lowland Maya Civilization. PNAS 109:3652-3657.

Eppich, Keith

2012 Lineage and State at El Perú-Waka': Ceramic and Architectural Perspectives on the Classic Maya Social Dynamic. Tesis doctoral, Department of Anthropology, Southern Methodist University, Dallas.

Escobedo, Héctor L.

1993 Entidades políticas del noroeste de las Montañas Mayas durante el período Clásico tardío. En VI Simposio de Investigaciones Arqueológicas en Guatemala, 1992, editado por Juan Pedro Laporte, Héctor Escobedo y Sandra Villagrán de Brady, pp. 1-21. Museo Nacional de Arqueología y Etnología, Ciudad de Guatemala.

Graham, Ian

1980 Corpus of Maya Hieroglyphic Inscriptions, Volume 2, Part 3: Ixkun, Ucanal, Ixtutz, Naranjo. Peabody Museum of Archaeology and Ethnology, Harvard University, Cambridge, Massachusetts.

Halperin, Christina T. y Jose Luis Garrido

2020 Architectural Aesthetics, Orientations, and Reuse at the Terminal Classic Maya Site of Ucanal, Petún, Guatemala. Journal of Field Archaeology 45:46-66.

Houston, Stephen D.

2008 In the Shadow of a Giant. Mesoweb. Documento electrónico, www.mesoweb.com/zotz/articles/Shadowof-a-Giant.pdf, accedido el 24 de agosto de 2021.

Houston, Stephen D., David Stuart y Karl Taube

2006 The Memory of Bones: Body, Being, and Experience among the Classic Maya. University of Texas Press, Austin.

Kaufman, Terence S. y William M. Norman

1984 An Outline of Proto-Cholan Phonology, Morphology and Vocabulary. En Phoneticism in 
Maya Hieroglyphic Writing, editado por John S. Justeson y Lyle Campbell, pp. 77-166. Institute for Mesoamerican Studies 9. State University of New York, Albany.

Lacadena García-Gallo, Alfonso

2010 Historia y ritual dinásticos en Machaquilá (Petén, Guatemala). Revista Española de Antropología Americana 41:205-240.

Laporte, Juan Pedro

1992 Los sitios arqueológicos del valle de Dolores en las montañas mayas de Guatemala. Mesoamérica 24:413-439.

Laporte, Juan Pedro y Héctor L. Escobedo

2009 Ixtutz: Algunas consideraciones generales. En Ixtutz y Curucuitz: Entidades políticas de la cuenca alta del río Poxte, Dolores y Poptun, Petén, editado por Juan Pedro Laporte, pp. 44-56. Monografía 8. Atlas Arqueológico de Guatemala, Dirección General de Patrimonio Cultural y Natural, Guatemala.

Laporte, Juan Pedro, con la colaboración de Héctor L. Escobedo y Phil Wanyerka

2009 Los monumentos esculpidos de Ixtutz y algunos aspectos históricos del sitio. En Ixtutz y Curucuitz: Entidades políticas de la cuenca alta del río Poxte, Dolores y Poptun, Petén, editado por Juan Pedro Laporte, pp. 116-163. Monografía 8. Atlas Arqueológico de Guatemala, Dirección General de Patrimonio Cultural y Natural, Guatemala.

Laporte, Juan Pedro y Héctor Mejía

2002 Tras la huella del Mopan: Arquitectura del Clásico terminal y del Postclásico en el sureste de Petén. En XV Simposio de Investigaciones Arqueológicas en Guatemala, 2001, editado por Juan Pedro Laporte, Héctor Escobedo y Bárbara Arroyo, pp. 59-88. Museo Nacional de Arqueología y Etnología, Ciudad de Guatemala.

2005 Los monumentos esculpidos de Ixkun y algunos aspectos históricos del sitio, con la colaboración de Héctor L. Escobedo y Phil Wanyerka. En Ixkun, Petén, Guatemala: Exploraciones en una ciudad del alto Mopan, 1985-2005, editado por Juan Pedro Laporte y Héctor Mejía, pp. 156-217. Atlas Arqueológico de Guatemala, Dirección General del Patrimonio Cultural y Natural, Ministerio de Cultura y Deportes, Guatemala.

2006 Exploraciones en el Grupo Oeste del área central de Sacul: Las Plazas A y B. En Sacul, Petén, Guatemala: Exploraciones en una entidad política de las Montañas Mayas, 1985-2006, editado por Juan Pedro Laporte y Héctor Mejía, pp. 22-67. Atlas Arqueológico de Guatemala, Dirección General del Patrimonio Cultural y Natural, Ministerio de Cultura y Deportes, Guatemala.

Laporte, Juan Pedro y Heidy Quezada

1998 Un acercamiento al postclásico en el sureste de Petén. En XI Simposio de Investigaciones Arqueológicas en Guatemala, 1997, editado por Juan Pedro Laporte y Héctor Escobedo, pp. 847-877. Museo Nacional de Arqueología y Etnología, Ciudad de Guatemala.

Laughlin, Robert, con John B. Haviland

1988 The Great Tzotzil Dictionary of Santo Domingo Zinacantán, with Grammatical Analysis and Historical Commentary, Volume I: Tzotzil-English. Smithsonian Institution, Washington, DC.

Los Angeles County Museum of Art

2020 Fragment of Carved Panel or Bench. Documento electrónico, http://collections.lacma.org/node/2260086, accedido el 23 de diciembre de 2020.
Maca, Allen L.

2002 Spatio-Temporal Boundaries in Classic Maya Settlement Systems: Copan's Urban Foothills and the Excavations at Group 9J-5. Tesis doctoral, Department of Anthropology, Harvard University, Cambridge, Massachusetts.

MacLeod, Barbara

1990 Deciphering the Primary Standard Sequence. Tesis doctoral, Department of Anthropology, University of Texas, Austin.

2022 Celebrations in the Heart of the Mountain. Bulletin of the Association for Mexican Cave Studies, en prensa.

Macri, Martha J. y Matthew G. Looper

2003 The New Catalog of Maya Hieroglyphs, Volume 1: The Classic Period Inscriptions. University of Oklahoma Press, Norman.

Martin, Simon y Nikolai Grube

2008 Chronicle of the Maya Kings and Queens: Deciphering the Dynasties of the Ancient Maya. 2da. ed. Thames and Hudson, Nueva York.

Martin, Simon y Joel Skidmore

2012 Exploring the 584,286 Correlation between the Maya and European Calendars. PARI Journal 13(2):316.

Mathews, Peter

1976 The Emblem Glyphs of Ucanal, Sacul, and Ixtutz. Maya Glyph Notes 2. Yale University, New Haven.

Mayer, Karl Herbert

1978 Maya Monuments: Sculptures of Unknown Provenance in Europe. Traducido por Sandra L. Brizee. Acoma Books, Ramona, California.

Mead, Julia C.

2003 ART: It's the End of the Road for a Treasured Museum. New York Times, el 22 de junio de 2003, p. 14.

Newman, Sarah, Jose Luis Garrido López y Nicholas P. Carter

2018 Collapse, Continuity, Change: El Zotz in the Terminal Classic Period. En An Inconstant Landscape: The Archaeology of El Zotz, Guatemala, editado por Stephen D. Houston, Thomas Garrison y Edwin Román Ramírez, pp. 116-139. University Press of Colorado, Boulder.

Reents-Budet, Dorie, Joseph W. Ball, Ronald L. Bishop, Virginia M. Fields y Barbara MacLeod

1994 Painting the Maya Universe: Royal Ceramics of the Classic Period. Duke University Press, Durham, North Carolina.

Ritter, Carl

1853 Über neue Entdeckungen und Beobachtungen in Guatemala und Yucatan. Zeitschrift für Ethnologie $1: 161-193$.

Robertson, Merle Greene

1972 Notes on the Ruins of Ixtutz, Southeastern Peten. Contributions of the University of California Archaeological Research Facility 16:89-104.

Smith, Robert E.

1955 Ceramic Sequence at Uaxactun, Guatemala. 2 vols. Middle American Research Institute, Tulane University, New Orleans; Carnegie Institution of Washington, Washington, DC.

Stuart, David S.

2006 The Language of Chocolate: References to Cacao on Classic Maya Drinking Vessels. En Chocolate in Mesoamerica: A Cultural History of Cacao, editado por Cameron L. McNeil, pp. 184-201. University Press of Florida, Gainesville. 
Tate, Carolyn

2013 Summer Solstice Ceremonies Performed by Bird Jaguar III of Yaxchilan, Chiapas, Mexico. Estudios de Cultura Maya 16:85-112.

Thompson, John Eric Sidney

1962 A Catalog of Maya Hieroglyphs. University of Oklahoma Press, Norman.

Van Swieten, G.

1976 Art de Mesoamerique/Meso-Amerikaanse Kunst. Catálogo de exhibición, el 17 de noviembre de 1976 al 8 de enero de 1977. Société Générale de Banque, Bruselas.
Webster, David

1989 The House of the Bacabs: Its Social Context. En The House of the Bacabs, Copan, Honduras, editado por David Webster, pp. 5-40. Studies in Pre-Columbian Art and Archaeology 29. Dumbarton Oaks, Washington, DC. Zender, Marc

2002 A Note on the Inscription of Ixtutz Stela 4. PARI Journal 2(4)/3(1):17-27.

Submitted February 20, 2021; Revised July 16, 2021; Accepted August 9, 2021 